\title{
Downregulated miR-1271-5p inhibits proliferation and invasion of trophoblast cells by activates Grhl2/CHL1 axis in preeclampsia
}

\section{Xue-Yan Shen}

The Second Hospital of Hebei Medical University

\section{Li-Li Cheng}

The Fourth Hospital of Shijiazhuang City

Jing Huang

The Second Hospital of Hebei Medical University

Hong-Fang Kong

The Second Hospital of Hebei Medical University

\section{Ya-Jing Chang}

The Second Hospital of Hebei Medical University

\section{Fang Wang}

The Second Hospital of Hebei Medical University

Hong Xin ( $\nabla$ xinhong_hb2h@163.com )

The Second Hospital of Hebei Medical University

\section{Research Article}

Keywords: preeclampsia, proliferation, invasion, Grhl2, miR-1271-5p, CHL1

Posted Date: November 3rd, 2021

DOI: https://doi.org/10.21203/rs.3.rs-926793/v1

License: (c) (1) This work is licensed under a Creative Commons Attribution 4.0 International License. Read Full License 
Downregulated miR-1271-5p inhibits proliferation and invasion of trophoblast cells by activates Grhl2/CHL1 axis in preeclampsia

Xue-Yan Shen ${ }^{1,2}$, Li-Li Cheng ${ }^{2}$, Jing Huang ${ }^{1}$, Hong-Fang Kong ${ }^{1}$, Ya-Jing Chang ${ }^{1}$, Fang Wang $^{1}$, Hong Xin ${ }^{1 *}$

(1.Department of Obstetrics, The Second Hospital of Hebei Medical University, Shijiazhuang 050000, P.R. China; 2. Department of Obstetrics, The Fourth Hospital of Shijiazhuang City, Shijiazhuang 050000, P.R. China)

* Corresponding Author: Pro. Hong Xin, Department of Obstetrics, The Second Hospital of Hebei Medical University, No.215, Heping West Road, Shijiazhuang 050000, Hebei Province, P.R. China; Tel.: +86-15803210560; E-mail: xinhong_hb2h@163.com

Authors' E-mail:

Xueyanshen_4hSJZ@163.com

lilizheng2008_2h@163.com

jinghuang_2013@163.com

hongfang_kong2010@126.com

yajing_chang2h@126.com

hbfangwang_2h@sina.com 


\begin{abstract}
Objective: Abnormal cell growth and invasion are known to be involved in the pathogenesis of preeclampsia (PE). Previous studies have shown that miR-1271-5p promotes cell proliferation and migration. However, the expression and function of miR-1271-5p in PE still remains unclear.

Materials and Methods: The expression of miR-1271-5p was detected from blood serum from pregnant and placental tissues. Silence or overexpression of miR-1271-5p in HTR8/SVneo cells. Real-time quantitative PCR was used to detect miR-1271-5p expression. Cell proliferation and invasion were determined using separately MTT assay Wound-scratch healing assay.
\end{abstract}

Results: In this study, we identified a downregulation in miR-1271-5p in blood samples and placentas of PE patients compared to healthy pregnant controls. In addition, overexpression of miR-1271-5p promoted trophoblast cell proliferation and invasion, while depletion of miR-1271-5p reduced these effects. Importantly, we revealed that grainyhead-like protein 2 homolog (Grhl2), which could inhibit proliferation and migration of trophoblast cells, was a direct target gene of miR-1271-5p in primary trophoblast cells and HTR-8/SVneo cells. Furthermore, Our results showed that Grhl2 bound to the cell adhesion molecule L1-like protein (CHL1) promoter and regulated it transcription in trophoblast cells.

Conclusion: Grhl2-mediated effects of miR-1271-5p on cell invasion and proliferation of trophoblast cells by promoting CHL1 transcription. The miR-1271-5p/Grhl2/CHL1 axis potentially provides a new therapeutic target for PE.

Key words: preeclampsia; proliferation; invasion; Grhl2; miR-1271-5p; CHL1 


\section{Introduction}

Pre-eclampsia (PE) is a specific disease of women during pregnancy characterized by proteinuria, high blood pressure, or renal insufficiency ${ }^{1}$. Approximately $5 \%$ of pregnant women will be affected by it. ${ }^{2}$. PE is the main cause of fetal morbidity and pregnancy death of women. Hypertension and proteinuria are the main clinical features of PE after 20 weeks of pregnancy in most women with normal blood pressure before pregnancy ${ }^{3-5}$. The pathogenesis of PE during pregnancy is mainly reduced or even lack of remodeling of the uterine spiral artery and insufficient oxygen supply to the placenta, and these changes are caused by a large number of excessive trophoblast cell apoptosis and partial invasion of superficial trophoblast cells 6, 7. Many factors affecting the occurrence and development of PE have been discovered, however, the clear molecular pathogenesis of PE is still largely unknown.

Understanding the genetic and epigenetic alterations involved in trophoblast cell behaviors would help in the development of novel therapeutic targets for the treatment of PE.

MicroRNAs (miRNAs) are a type of non-coding RNA with a size of $\sim 22 \mathrm{nt}$, which regulate the expression of genes at the post-transcription level and play a vital role in biological processes ${ }^{8,9}$. Research by Zhao JR and Chang $G$ et al. reported that miRNAs regulate the growth, apoptosis, invasion and migration of trophoblast cells and play an important role in the development of the placenta ${ }^{10,11}$. A research report proved that reducing the expression of miR-221-3p during PE inhibits the growth, invasion and migration of trophoblasts by targeting the expression of THBS $2^{12}$. Another study reported that miR-454 targets the expression of 3'UTR of EPHB4 to promote the proliferation and invasion of trophoblast cells ${ }^{13}$. Recent studies showed that miR-1271-5p plays an important role in the development of type 1 and type 2 diabetes by promoting cell proliferation and migration ${ }^{14,15}$. However, the expression and function of miR-1271-5p in PE is still unclear.

Grhl2, an important wound healing transcription factor, has been extensively studied in cell metastasis and proliferation including oral cancer, non-small cell lung cancer, gastric cancer, and ovarian cancer ${ }^{16,17}$. Study reported that inhibition of the Grhl2 gene inhibited proliferation, migration, invasion, and mediation of cell cycle arrest ${ }^{18}$. Loss of Grhl2 also inhibits proliferation and colony formation of cells ${ }^{19}$. Moreover, the expression of Grhl2 is also regulated by miRNA at the posttranscriptional level. It has been reported that miR-217 and miR-194 directly target the Grhl2 3'UTR and negatively regulates Grhl2 levels in keratinocytes ${ }^{20,21}$. A recent study has shown that Grhl2 was highly expressed in chorionic trophoblast cells, including basal chorionic trophoblast cells located at the mouse placental chorioallantoic fluid interface ${ }^{22}$. Our previous research found a circTRNC18 inhibited trophoblast cell migration and epithelial-mesenchymal transition by regulating miR-762/Grhl2 pathway in PE ${ }^{23}$. However, the regulation and function of Grhl2 in PE pathogenesis remains largely unknown. 
In our current study, we have confirmed that miR-1271-5p is significantly downregulated in PE blood samples and placental tissue. Overexpression of miR-1271-5p promoted while depletion of it inhibited trophoblast cell proliferation and invasion. Mechanistically, miR-1271-5p binds to Grhl2 3'UTR and regulates Grhl2 expression. Grhl2-mediated effects of miR-1271-5p on cell invasion and proliferation of trophoblast cells by promoting CHL1 transcription.

\section{Materials and methods}

\section{Clinical samples}

30 pairs of PE and normal placentas were collected from the pregnancy women who gave birth at the Department of Obstetrics, The forth hospital of Shijiazhuang, from July 2016 to October 2019 23 . PE diagnosis was according to ACOG 2013 guideline ${ }^{23}$. Tissue were collected from the mother surface of the placenta as well as near the root of the umbilical cord. The study protocol was approved by the Ethics Committee of Second Hospital of Hebei Medical University and written consent was obtained from each participant. All patients provided written informed consent.

\section{Cell culture and transfection}

Human extravillous trophoblast cell line HTR8/SVneo were maintained in our laboratory and primary human trophoblasts were isolated from normal term placentas as described previously ${ }^{24,25}$. Cells were cultured in RPMI 1640 medium (Gibco) supplemented with $10 \%$ FBS, 100 units/ml penicillin and $100 \mu \mathrm{g} / \mathrm{ml}$ streptomycin and the two antibiotics listed above. Cell transfection was following Lipofectamine 2000 (Invitrogen) manufacturer's protocol. The miR-1271-5p mimics, mimic-NC, miR-1271-5p inhibitor, inhibitor-NC were purchased from GenePharma Co., Ltd (Shanghai, China). Grhl2 overexpression vector, shCHL1 and CHL1 luciferase reporter vector were purchased from GENEWIZ Company (Suzhou, China).

\section{Transwell invasion assay}

$8-\mu \mathrm{m}$ pore size transwell filters (Costar) were coated with a matrigel (Sigma-Aldrich) prior to plating cells ${ }^{23} .3 \times 10^{4}$ cells/well HTR-8/SVneo cells were seeded on the upper chambers with serum-free medium ${ }^{23}$. After culturing at $37{ }^{\circ} \mathrm{C}$ and $5 \% \mathrm{CO}_{2}$ for 24 hours, cells with higher migration ability on the upper side of the chamber were migrated into the lower chamber ${ }^{23}$. The migration cells number was counted in 3 randomly selected areas under a light 
microscope.

\section{Western blot analysis}

RIPA buffer was used for lysing cultured cells. Then the proteins were running on $8 \%-10 \%$ SDS-PAGE, and electro-transferred to a PVDF membrane (Millipore). After blocking in 5\% milk in TTBS, the membranes were incubated with primary antibodies at. Then the blots were detected by ECL (enhanced chemiluminescence) Fuazon Fx (Vilber Lourmat) Antibodies that were used are as follow: anti-Grhl2 (1:1000, ab86611), anti- $\beta$-catenin (1:1000, 51067-2-AP), anti-CHL1 (1:1000, 25250-1-AP) and anti- $\beta$-actin (1:1000, sc-47778). Images were captured and processed by FusionCapt Advance Fx5 software (Vilber Lourmat). All experiments were replicated three times independently. The description of this method refers to another article by this author ${ }^{23}$.

\section{Luciferase assay}

HTR-8/SVneo cell were seeded in 24 well plates. HTR-8/SVneo cells were co-transfected with a miR-1271-5p mimic or NC mimic (200 pmol) and CHL1-luciferase reporter or an empty vector together; For miR-1271-5p-Grlh2 luciferase assay, HTR-8/SVneo cells were transfected with a miRNA-1271 mimic or NC mimic combined with Grhl2-3'UTR-luciferase reporter (wt or mut). A Dual-Glo Luciferase Assay System (Promega) with a Flash and Glow (LB955) was used to measure Luciferase activity. The description of this method refers to another article by this author ${ }^{23}$.

\section{RNA extraction and quantitative real-time PCR}

QIAzol Lysis Reagent (\#79306) was used for lysing tissues and cultured cells. NanoDrop 2000 (Thomer Fisher) was used for exam the purity and quality of RNA. microRNA reverse transcription was used the miScripIIRT kit (QIAGEN) following its protocol. miScript SYBR ${ }^{\circledR}$ Green PCR kit was used for qRT-PCR of miRNA a with following primers : miR-1271-5p: GGC TTG GCA CCT AGC AAG CAC TC; U6-F:GTG CTC GCT TCG GCA GCA CAT ATAC; U6-R:AAA ATA TGG AAC GCT TCA CGA ATT TGC.. M-MLV First Strand Kit (Life Technologies)was used reverse transcription of mRNA and circRNA. qRT-PCR of mRNA and circRNA was according to the protocol of platinum SYBR Green qPCR Super Mix UDG Kit (Invitrogen). CFX96 ${ }^{\mathrm{TM}}$ Real-Time System (Bio-Rad) was used to preform qRT-PCR experiments with primers of Grhl2-F: CCG GCT GCT GCT ACT ACT AC; Grhl2-R: GCA CTC GGT TTT CTC CTC CA. GAPDH-F: CAC CAT CTT CCA GGA GCG AG; GAPDH-R: GAC TCC ACG ACG TAC TCA GC. TEAD1-F: 
AACTCAGGACAGGCAAGACG; TEAD1-R: GGCTTGACGTCTTGTGAGGA; S1PR2-F:TCCTGCCCGATCCTCTACAA； S1PR2-R:GAGAAGATGGTCACCACGCA； CYLD-F:AAACATGCTTTGGGACTGCC；CYLD-R:TGAATGCCCCACAGAACGAT ; NR4A3-F:CTTTGCAACGCTGACGGTG； NR4A3-R:GGGGAAGGGCTATATTGGGC；

ISL1-F: CTTTCAGCATTGGCAACCCC; ISL1-R: ACACAGCGGAAACACTCGAT; PAK2-F:TTGCCCGGGGCCATTTCATAA; PAK2-R:GTGCTGCTCATTCGCACAG; Sgk3-F: CGGGGCCAGGTTGCAT; Sgk3-R: GTGTTCATCGGAGCTGGGAA; PAK1-F: GTGGTGGCTGCACAGTAGA; PAK1-R: GGCTCCGTGGAAAAGGGAAT; MOB1B-F: TTCTTGTTGAGTTCTCAGCCT; MOB1B-R: ATCCAACTGGTCCTGAACCC; All data were analyzed by adopting $2^{-\Delta \Delta} \mathrm{Ct}$ method. The description of this method refers to another article by this author ${ }^{23}$.

\section{MTS assay}

MTS assay was performed as previously described ${ }^{26}$. In brief, the medium of cultured HTR-8/SVneo was replaced with $100 \mu \mathrm{l}$ serum-free RPMI 1640 containing $10 \mu 1$ of CellTiter 96 AQueous One Solution (Promega). Control reactions were performed in triplicate using the same reagents without cells. The plates were then incubated at $37^{\circ} \mathrm{C}$ for 4 hours. Then, 60 $\mu \mathrm{l}$ of medium from each well was transferred to a new 96-well plate, and the absorbance was at $490 \mathrm{~nm}$ was measured using a Multiskan spectrum (Thermo). The control absorbance at $490 \mathrm{~nm}$ was subtracted from the average absorbance to obtain the corrected absorbance.

\section{Target prediction}

For finding the potential target of miR-1271-5p, we used the miRanda ${ }^{27}$ (www.microrna.org), RNAhybrid 28 (http://bibiserv.techfak.uni-bielefeld.de/rnahybrid/submission.html) and Targetscan $^{29}$ (http://www.targetscan.org/vert_72/).

\section{Wound-scratch healing assay}

trophoblast cell grown on 12-well plates were co-transfected with pcDNA3.1-Grhl2, control vector. Confluent cells were scraped using $200-\mu \mathrm{L}$ pipette tips, washed twice with phosphate-buffered saline, and then cultured with $2 \%$ serum media for 24 hours. Images were acquired using a CKX41 microscope (Olympus Corporation, Tokyo, Japan) equipped with an EOS 600D camera (Canon, Tokyo, Japan) immediately at 0 and 24 hours after the wounding. The description of this method refers to another article by this author ${ }^{23}$.

\section{Statistical analysis}


Data are presented as mean \pm SEM. The differences between two groups were analyzed by Student's t test. Spearman's correlation analysis was used for evaluating the correlation between circRNA and miRNA. $P<0.05$ were considered statistically significant. Statistical analysis was performed using Graphpad Prism 7.0 software (GraphPad Software, CA, USA). The description of this method refers to another article by this author ${ }^{23}$.

\section{Results}

\section{Clinical characteristics of pregnant women with PE and healthy pregnant controls}

To begin with, we studied the clinical characteristics of PE patients and compared them to our healthy pregnant control group. We found no significant difference in the maternal age and pre-pregnancy body mass index between women with $\mathrm{PE}$ and healthy pregnant controls (Table 1). Patients with PE showed increased systolic blood pressure $(P<0.001)$ and diastolic blood pressure $(P<0.001)$ compared with healthy pregnant controls. The patients with $\mathrm{PE}$ also had proteinuria, while proteinuria was not detectable in the normal pregnant controls (Table 1). The patients with PE also had a lower gestational age at delivery and lower birth weight of baby, but no difference was found regarding baby gender compared with healthy pregnant controls (Table 1).

\section{miR-1271-5p is decreased in serum and placental tissues from pregnant women with $P E$}

Previous studies have shown that miR-1271-5p plays an important role in cell proliferation and migration ${ }^{30,31}$. To study whether miR-1271-5p plays a role in the pathogenesis of PE, we first analyzed the expression of miR-1271-5p in the blood serum from pregnant women by RT-qPCR.. Our results showed that the expression of miR-1271-5p was significantly downregulated in the PE group compared with the normal pregnancy control group (Fig. 1A). To confirm this, we further examined miR-1271-5p levels in placental tissues. As expected, the expression of miR-1271-5p was also markedly downregulated in placental tissues in the PE group compared to the normal pregnancy group (Fig. 1B). These data suggest that decreased miR-1271-5p may contribute to the progression and development of PE.

\section{miR-1271-5p plays a role in the proliferation and invasion of trophoblast cells}

Because the abnormal growth and invasion of trophoblast cells is associated with PE pathogenesis, we want to investigate if miR-1271-5p was correlated with trophoblast cell 
proliferation and invasion. To do this, we firstly transfected HTR8/SVneo cells with the miR-1271-5p mimic, miR-1271-5p inhibitor, and their negative controls, respectively. As shown in Fig. 2A, transfection with miR-1271-5p in HTR8/SVneo cells significantly upregulated the miR-1271-5p level compared with control mimic detection by RT-qPCR analysis. On the contrary, miR-1271-5p inhibitor transfection led to a dramatic downregulation of the miR-1271-5p level compared to the control inhibitor (Fig. 2B). We then assessed the role of miR-1271-5p in the regulation of proliferation of trophoblast cells. Our results showed that miR-1271-5p overexpression significantly increased proliferation of HTR-8/SVneo cells, while miR-1271-5p knockdown exhibited opposite effects (Fig. 2C). Subsequently, transwell assays were performed to detect the effect of miR-1271-5p on HTR8/SVneo cell invasion. miR-1271-5p mimic transfection significantly promoted the invasion ability of HTR-8/SVneo cells (Fig. 2D and E). In contrast, the depletion of miR-1271-5p levels resulted in a decreased ability of invasion in HTR-8/SVneo cells compared to negative controls (Fig. 2F and G). These findings suggest that miR-1271-5p may be responsible for trophoblast cell proliferation and invasion.

\section{Grhl2 is a target of miR-1271-5p in trophoblast cells}

Because miRNAs can regulate gene expression by binding to the mRNA 3'UTR. Thus, we analyzed the potential targets of miR-1271-5p by using three target prediction programs, including miRDB, RNA22 and TargetScan. A total of 66 predicted genes may be miR-1271-5p target genes, and only 11 of them are related to cell proliferation and migration (Fig. 3A). Secondly, we transfected HTR8/SVneo cells with the miR-1271-5p mimic or inhibitor, and their negative controls, and then used RT-qPCR to detect these candidates genes expression. The results showed that only Grhl2 downregulation in miR-1271-5p mimic and upregulation in miR-1271-5p inhibitor transfected- HTR8/SVneo cells (Fig. 3B). Analysis showed that mRNA 3'-UTR of Grhl2 has a conserved binding sites for miR-1271-5p seed regions (Fig. 3C). However, whether or not miR-1271-5p binds to Grhl2 has not been investigated. We then co-transfected miR-1271-5p mimic and Grh2-3'-UTR-luciferase reporter in HTR-8/SVneo cells. The luciferase analysis showed that miR-1271-5p mimic significantly decreased luciferase activity mediated by wild-type 3'-UTR; mutation of the miR-1271-5p-binding site did not affect luciferase activity in the presence of the miR-1271-5p mimic (Fig. 3D). Next, we examined whether or not miR-1271-5p directly regulates the expression of miR-1271-5p in trophoblast cells. As shown in Fig. 3E and F, overexpression of miR-1271-5p markedly decreased the protein level of Grhl2, whereas depletion of miR-1271-5p by transfecting with a miR-1271-5p inhibitor markedly elevated Grhl2 expression. Additionally, we further isolated and investigated Grhl2 expression in 
primary trophoblast cells after transfection of miR-1271-5p mimic and inhibitor. Our results revealed that miR-1271-5p also negatively regulated Grhl2 expression in primary trophoblast cells (Fig. 3G, H and I). These data reveal that miR-1271-5p directly targets the 3'-UTR of Grhl2 and regulates it expression.

\section{Grhl2 inhibits the proliferation and migration of trophoblast cells}

Prior reports indicate that Grhl2 slows down multicells proliferation and migration ${ }^{23,32}$, and the above results indicate that the expression of Grhl2 in PE tissues is increased. We tried to determine whether cell proliferation and migration are regulated by Grhl2 at this stage of the study. We transfected the Grhl2 overexpression vector in trophoblast cells and the results showed that Grhl2 mRNA and protein expression levels were significantly upregulated (Figs. 4A-4C). Then, we used the MTS test to detect the proliferation of the cells, and the results showed that compared with the vector control group, the overexpression of Grhl2 significantly slowed down the proliferation of trophoblast cells (Fig. 4D). Addition, the results of the wound scratch healing test also proved that overexpression of Grhl2 hinders the migration of trophoblast cells (Figs. 4E and F). These results support that Grhl2 overexpression inhibits the proliferation and migration of trophoblast cells.

\section{Grhl2-mediated effects of miR-1271-5p on cell invasion and proliferation of trophoblast cells}

To further understand the relationship between miR-1271-5p and Grhl2 in PE, we initially examined Grhl2 expression in placental tissues from PE patients. As shown in Fig.5A, the expression of Grhl2 was markedly upregulated in placental tissues from PE patients compared to that in normal placental tissues. We further analyzed the correlation of miR-1271-5p and Grhl2, and found that Grhl2 expression was negatively correlated with miR-1271-5p expression in placental tissues from PE patients (Fig. 5B). To verify if the function of miR-1271-5p on trophoblast cells is exerted via regulating Grhl2, we performed rescue experiments by co-transfecting the Grhl2 expression vector and miR-1271-5p mimic. Transwell assay showed that overexpression of Grhl2 in HTR-8/SVneo cells significantly depressed cell invasion compared to control vector and also reversed the inhibitory effect of miR-1271-5p mimic transfection on cell invasion (Fig. 5C and D). Similarly, an overexpression of Grhl2 attenuated the effects of miR-1271-5p overexpression on cell growth (Fig. 5E). Taken together, these data indicate that Grhl2 is involved in miR-1271-5p-mediated trophoblast cell proliferation and invasion. 
miR-1271-5p promotes proliferation and invasion of trophoblast cells by depresses Grhl2/CHL1 axis

Previous studies have shown that loss of CHL1 promotes tumor growth and metastasis in esophageal squamous cell carcinoma ${ }^{33}$. However, whether Grhl2 inhibits migration and invasion of trophoblast cells via regulates CHL1. In order to verify this, we performed rescue experiments by co-transfecting the Grhl2 vector and miR-1271-5p mimic. As showed in Fig. 6A and B, miR-1271-5p mimic transfection significant reduced the CHL1 but not $\beta$-catenin protein level. However, overexpression of Grhl2 promoted the expression of CHL1 regardless miR-1271-5p mimic transfection or not. This result indicated that miR-1271-5p regulated CHL1 expression dependent on Grhl2. Next, we applied the Ensembl and Promo 3.0 website approaches to search for the potential binding site of Grhl2 on the 2-kb--promoter region of CHL1 and found 3 potential binding elements within this region (Fig. 6C). Then, we used a luciferase reporter assay to detect whether CHL1 is a direct transcriptional target of Ghrl2. The luciferase assay revealed that the proximal 2000-bp promoter region (1279 to $1227 \mathrm{bp}$ upstream of the transcription start site) is essential for the Grhl2-mediated transcription of CHL1 (Fig. 6D). Furthermore, ChIP analysis confirmed that Grhl2 bound predominantly to the proximal region of the CHL1 promoter in trophoblast cells (Fig. 6E), suggesting that CHL1 is a direct target of Grhl2. Then, using MTS assay we found that overexpressing Grhl2 significantly inhibits trophoblast cells proliferation while knocked down of CHL1 revised these effects (Fig. 6F). Together, these data indicate that miR-1271-5p regulates proliferation and invasion of trophoblast cells by suppressed Grhl2/CHL1 axis.

\section{Discussion}

miRNAs play an important role in many disease processes including PE ${ }^{34}$. In this study, we revealed that: 1) miR-1271-5p is decreased in placental tissues and serum from pregnant women with PE; 2) overexpression of miR-1271-5p promotes trophoblast cells proliferation and invasion; 3) miR-1271-5p directly targets Grhl2 3'UTR depressing its expression in trophoblast cells; 4) Grhl2-mediated effects of miR-1271-5p on cell invasion and proliferation of trophoblast cells, thus regulating PE progression; 5) miR-1271-5p regulated proliferation and invasion of trophoblast cells by depresses Grhl2/CHL1 axis.

It is generally believed that the invasiveness of trophoblastic cells in the uterine spiral artery has a key role in PE pathogenesis. Trophoblasts are the main components of the placental structure and play an important role in placental formation and the maintenance of normal fetal development ${ }^{35}$. During placenta formation, trophoblast invasion occurs, narrowing the spiral artery, thus reducing placental blood flow ${ }^{36}$. On the one hand, it can 
cause intrauterine growth restriction. On the other hand, trophoblasts secrete certain cytokines or hormones, which are circulated around the entire body ${ }^{37}$. Damage to vascular endothelial function and inflammatory reaction are excessive, causing a series of clinical symptoms such as hypertension and proteinuria, thereby inducing $\mathrm{PE}^{38,39}$. This process involves complex cell signal transduction pathways, but the specific molecular mechanism has not yet been fully elucidated. Our previous study found that a circTNRC18 was significantly upregulated in PE placentas and circTNRC18 affected PE progression by inhibiting trophoblast cells EMT and migration. In this study, we found that the expression of miR-1271-5p was significant downregulated in PE tissues. The downregulation of miR-1271-5p promoted level of Grhl2 which can inhibit both invasion and proliferation of trophoblast cells and accelerate PE progression. However, why the expression of miR-1271-5p was down-regulated in PE needs to be investigated further.

Grhl2 is a member of the grainy head-like transcription factor family and a homolog of Drosophila grainy head in mammals ${ }^{40}$. Studies have shown that family members play an important role in growth and development, neural tube closure, epithelial cell differentiation, and cell proliferation and migration ${ }^{41,42}$. Although Walentin $\mathrm{K}$ et al. found in animal studies that the Grhl2-dependent gene network controls trophoblastic branch morphogenesis, the role of Grhl2 in the PE process remains unclear ${ }^{22}$. The placenta from Grhl2-deficient mouse embryos showed defects in BCT cell polarity and basement membrane integrity at the chorioallantoic membrane interface ${ }^{43}$. Grhl2 depresses cell migration by inhibiting EMT of trophoblast cells ${ }^{23}$. Report shown that Grhl2 reduced the protein expression of matrix metalloproteinase-2, -7 and -9 (MMP-2, MMP-7 and MMP-9) ${ }^{44}$. Those MMPs play an important role in the progression of $\mathrm{PE}^{45,46}$. This study also found that Grhl2 is upregulated in PE tissues. Upregulated Grhl2 may promote the progression of PE by inhibiting the proliferation and migration of trophoblast cells. Grhl2-mediated effects of miR-1271-5p on cell invasion and proliferation of trophoblast cells by promoting CHL1 expression.

The cell adhesion molecule L1-like protein (CHL1) belongs to the transmembrane adhesion molecule of the immunoglobulin superfamily and exhibits biological functions involved in signal transduction pathways ${ }^{47,48}$. A great mount of studies shown that CHL1 played an important role in cell growth and metastasis ${ }^{49}$. Juan C. et al. reported that CHL1 suppresses tumor cell growth and metastasis by repressing PI3K/AKT signaling pathway ${ }^{49}$. CHL1 is downregulated by hypermethylation and that this epigenetic alteration is an independent prognostic factor and a potential biomarker of poor prognosis in breast cancer ${ }^{50}$. As an important tumor suppressor, CHL1 play the function with both anti-proliferation and antimetastasis abilities, through Merlin and SEMA3B-Np1-mediated inhibition of AKT signaling pathway ${ }^{33}$. However, the expression and function of CHL1 in PE still unclear. In 
our current study, we confirmed that the expression of CHL1 was upregulated in PE placental tissue. Mechanistically, downregulated miR-1271-5p promotes Grhl2 expression in trophoblast cells and accelerates PE progression. As a transcription factor, Grhl2 directly binding to CHL1 promoter and promotes CHL1 expression. The downregulation of miR-1271-5p inhibits proliferation and invasion of trophoblast cells by activates Grhl2/CHL1 axis.

Studies have shown that, throughout the pregnancy, the trophoblast cells always maintain a dynamic balance in order to ensure the supply of fetal nutrition. Our study has some limitations ${ }^{51}$. Firstly, we only analyzed the expressions of miR-1271-5p and Grhl2 in placental tissues and blood samples obtained from late gestation, which may not be the time that trophoblast invasion first took place. More early such as 20 weeks of pregnancy's blood samples of PE patients should be measured, and then compare them with blood samples in the late gestation. Second, more samples size and in vivo studies should be included in future study to confirm the findings.

In summary, miR-1271-5p is downregulated in PE blood and tissues. Functional assays showed that miR-1271-5p directly depressed Grhl2 expression by binding its 3'UTR. Grhl2-mediated effects of miR-1271-5p on cell invasion and proliferation of trophoblast cells by promoting CHL1 transcription. The miR-1271-5p/Grhl2/CHL1 axis potentially provides a new therapeutic target for PE.

\section{Declarations}

All methods involved in this article were carried out in accordance with relevant guidelines and regulations, and with ethical approval and consent.

\section{Acknowledgments}

The authors would like to thank The Second Hospital of Hebei Medical University (Shijiazhuang, China) for their assistance and cooperation during the present study.

\section{Funding}

This study was partially supported by Hebei Provincial Natural Science Foundation of China (H2020106239).

\section{Availability of data and materials}

The datasets used and/or analyzed during the present study are available from the corresponding author on reasonable request. 


\section{Authors' Contributions}

X.S and H.X conceived and designed the experiments. X.S and L.C performed the experiments. X.S and J.H analyzed the data. H.K and Y.C interpreted the data, and X.S wrote the first draft of the manuscript. X.S and H.X gave final approval of the results and conclusions made in the manuscript. F.W analyzed the results and developed the structure and arguments for the paper. X.S, L.C and J.H critically revised the paper for imporᄀtant intellectual content. All authors read and approved the final manuscript.

\section{Ethics approval and consent to participate}

All of the women enrolled in the present study provided written informed consent prior to inclusion to the study. The study protocol was approved by the Ethics Committee of Second Hospital of Hebei Medical University (approval number 2019126).

\section{Conflict of interest}

The authors declare that there are no conflicts of interest.

\section{References}

1. El-Sayed AAF. Preeclampsia: A review of the pathogenesis and possible management strategies based on its pathophysiological derangements. Taiwan J Obstet Gynecol. Oct 2017;56(5):593-598.

2. Ramos JGL, Sass N, Costa SHM. Preeclampsia. Rev Bras Ginecol Obstet. Sep 2017;39(9):496-512.

3. Tranquilli AL, Brown MA, Zeeman GG, Dekker G, Sibai BM. The definition of severe and early-onset preeclampsia. Statements from the International Society for the Study of Hypertension in Pregnancy (ISSHP). Pregnancy Hypertens. Jan 2013;3(1):44-47.

4. Phipps EA, Thadhani R, Benzing T, Karumanchi SA. Pre-eclampsia: pathogenesis, novel diagnostics and therapies. Nat Rev Nephrol. May 2019;15(5):275-289.

5. Nakanishi S, Aoki S, Nagashima A, Seki K. Incidence and pregnancy outcomes of superimposed preeclampsia with or without proteinuria among women with chronic hypertension. Pregnancy Hypertens. Jan 2017;7:39-43.

6. Saito $\mathrm{S}$, Nakashima A. A review of the mechanism for poor placentation in early-onset preeclampsia: the role of autophagy in trophoblast invasion and vascular remodeling. $J$ Reprod Immunol. Mar 2014;101-102:80-88.

7. Ji L, Brkic J, Liu M, Fu G, Peng C, Wang YL. Placental trophoblast cell differentiation: physiological regulation and pathological relevance to preeclampsia. Mol Aspects Med. Oct 2013;34(5):981-1023.

8. Kosik KS, Nowakowski T. Evolution of New miRNAs and Cerebro-Cortical Development. Annu Rev Neurosci. Jul 8 2018;41:119-137. 
9. Bartel DP. MicroRNAs: genomics, biogenesis, mechanism, and function. Cell. Jan 23 2004;116(2):281-297.

10. Zhao JR, Cheng WW, Wang YX, Cai M, Wu WB, Zhang HJ. Identification of microRNA signature in the progression of gestational trophoblastic disease. Cell Death Dis. Jan 24 2018;9(2):94.

11. Chang G, Mouillet JF, Mishima T, et al. Expression and trafficking of placental microRNAs at the feto-maternal interface. FASEB J. Jul 2017;31(7):2760-2770.

12. Yang $\mathrm{Y}$, Li H, Ma Y, Zhu X, Zhang S, Li J. MiR-221-3p is down-regulated in preeclampsia and affects trophoblast growth, invasion and migration partly via targeting thrombospondin 2. Biomed Pharmacother. Jan 2019;109:127-134.

13. Wang F, Yan J. MicroRNA-454 is involved in regulating trophoblast cell proliferation, apoptosis, and invasion in preeclampsia by modulating the expression of ephrin receptor B4. Biomed Pharmacother. Nov 2018;107:746-753.

14. Wang Y, Xu L, Jiang L. Retraction notice to "miR-1271 promotes non-small-cell lung cancer cell proliferation and invasion via targeting HOXA5" [Biochem. Biophys. Res. Commun. 458/3 (2015) 714-719]. Biochem Biophys Res Commun. Aug 6 2019;515(4):729.

15. Massaro JD, Polli CD, Costa ESM, et al. Post-transcriptional markers associated with clinical complications in Type 1 and Type 2 diabetes mellitus. Mol Cell Endocrinol. Jun 15 2019;490:1-14.

16. Morrison CD, Parvani JG, Schiemann WP. The relevance of the TGF-beta Paradox to EMT-MET programs. Cancer Lett. Nov 28 2013;341(1):30-40.

17. Frisch SM, Farris JC, Pifer PM. Roles of Grainyhead-like transcription factors in cancer. Oncogene. Nov 2 2017;36(44):6067-6073.

18. Faddaoui A, Sheta R, Bachvarova M, et al. Suppression of the grainyhead transcription factor 2 gene (GRHL2) inhibits the proliferation, migration, invasion and mediates cell cycle arrest of ovarian cancer cells. Cell Cycle. Apr 3 2017;16(7):693-706.

19. Chen W, Yi JK, Shimane T, et al. Grainyhead-like 2 regulates epithelial plasticity and stemness in oral cancer cells. Carcinogenesis. May 2016;37(5):500-510.

20. Yu X, An J, Hua Y, et al. MicroRNA-194 regulates keratinocyte proliferation and differentiation by targeting Grainyhead-like 2 in psoriasis. Pathol Res Pract. Feb 2017;213(2):89-97.

21. Zhu H, Hou L, Liu J, Li Z. MiR-217 is down-regulated in psoriasis and promotes keratinocyte differentiation via targeting GRHL2. Biochem Biophys Res Commun. Feb 26 2016;471(1):169-176.

22. Walentin K, Hinze C, Werth M, et al. A Grhl2-dependent gene network controls trophoblast branching morphogenesis. Development. Mar 15 2015;142(6):1125-1136.

23. Shen XY, Zheng LL, Huang J, et al. CircTRNC18 inhibits trophoblast cell migration and epithelial-mesenchymal transition by regulating miR-762/Grhl2 pathway in pre-eclampsia. $R N A$ Biol. Nov 2019;16(11):1565-1573.

24. Aboagye-Mathiesen G, Laugesen J, Zdravkovic M, Ebbesen P. Isolation and characterization of human placental trophoblast subpopulations from first-trimester chorionic villi. Clin Diagn Lab Immunol. Jan 1996;3(1):14-22.

25. Kliman HJ, Nestler JE, Sermasi E, Sanger JM, Strauss JF, 3rd. Purification, characterization, and in vitro differentiation of cytotrophoblasts from human term placentae. Endocrinology. Apr 1986;118(4):1567-1582. 
26. Yang Z, Zheng B, Zhang Y, et al. miR-155-dependent regulation of mammalian sterile 20-like kinase 2 (MST2) coordinates inflammation, oxidative stress and proliferation in vascular smooth muscle cells. Biochim Biophys Acta. Jul 2015;1852(7):1477-1489.

27. John B, Enright AJ, Aravin A, Tuschl T, Sander C, Marks DS. Human MicroRNA targets. PLoS Biol. Nov 2004;2(11):e363.

28. Rehmsmeier M, Steffen P, Hochsmann M, Giegerich R. Fast and effective prediction of microRNA/target duplexes. RNA. Oct 2004;10(10):1507-1517.

29. Agarwal V, Bell GW, Nam JW, Bartel DP. Predicting effective microRNA target sites in mammalian mRNAs. Elife. Aug 12 2015;4.

30. Li Q, Shi J, Xu X. MicroRNA-1271-5p inhibits the tumorigenesis of ovarian cancer through targeting E2F5 and negatively regulates the mTOR signaling pathway. Panminerva Med. May 14 2020.

31. Lin MF, Yang YF, Peng ZP, et al. FOXK2, regulted by miR-1271-5p, promotes cell growth and indicates unfavorable prognosis in hepatocellular carcinoma. Int J Biochem Cell Biol. Jul 2017;88:155-161.

32. Zhou B, Zhang X, Li T, et al. CircZDHHC20 represses the proliferation, migration and invasion in trophoblast cells by miR-144/GRHL2 axis. Cancer Cell Int. 2020;20:19.

33. Tang H, Jiang L, Zhu C, et al. Loss of cell adhesion molecule L1 like promotes tumor growth and metastasis in esophageal squamous cell carcinoma. Oncogene. Apr 2019;38(17):3119-3133.

34. Biro O, Nagy B, Rigo J, Jr. Identifying miRNA regulatory mechanisms in preeclampsia by systems biology approaches. Hypertens Pregnancy. Feb 2017;36(1):90-99.

35. Burton GJ, Jauniaux E. Pathophysiology of placental-derived fetal growth restriction. Am J Obstet Gynecol. Feb 2018;218(2S):S745-S761.

36. Moser G, Huppertz B. Implantation and extravillous trophoblast invasion: From rare archival specimens to modern biobanking. Placenta. Aug 2017;56:19-26.

37. Abbas Y, Oefner CM, Polacheck WJ, et al. A microfluidics assay to study invasion of human placental trophoblast cells. J R Soc Interface. May 2017;14(130).

38. Possomato-Vieira JS, Khalil RA. Mechanisms of Endothelial Dysfunction in Hypertensive Pregnancy and Preeclampsia. Adv Pharmacol. 2016;77:361-431.

39. Black KD, Horowitz JA. Inflammatory Markers and Preeclampsia: A Systematic Review. Nurs Res. May/Jun 2018;67(3):242-251.

40. Wilanowski T, Tuckfield A, Cerruti L, et al. A highly conserved novel family of mammalian developmental transcription factors related to Drosophila grainyhead. Mech Dev. Jun 2002;114(1-2):37-50.

41. Kang MK, Chen W, Park NH. Regulation of Epithelial Cell Proliferation, Differentiation, and Plasticity by Grainyhead-Like 2 During Oral Carcinogenesis. Crit Rev Oncog. 2018;23(3-4):201-217.

42. Mlacki M, Kikulska A, Krzywinska E, Pawlak M, Wilanowski T. Recent discoveries concerning the involvement of transcription factors from the Grainyhead-like family in cancer. Exp Biol Med (Maywood). Nov 2015;240(11):1396-1401.

43. Walentin K, Hinze C, Schmidt-Ott KM. The basal chorionic trophoblast cell layer: An emerging coordinator of placenta development. Bioessays. Mar 2016;38(3):254-265. 
44. Xiang J, Fu X, Ran W, Wang Z. Grhl2 reduces invasion and migration through inhibition of TGFbeta-induced EMT in gastric cancer. Oncogenesis. Jan 9 2017;6(1):e284.

45. Sahay AS, Jadhav AT, Sundrani DP, Wagh GN, Mehendale SS, Joshi SR. Matrix metalloproteinases-2 (MMP-2) and matrix metalloproteinases -9 (MMP-9) are differentially expressed in different regions of normal and preeclampsia placentae. J Cell Biochem. Aug 2018;119(8):6657-6664.

46. Cheng D, Liang B, Li YH. Application of MMP-7 and MMP-10 in assisting the diagnosis of malignant pleural effusion. Asian Pac J Cancer Prev. 2012;13(2):505-509.

47. He LH, Ma Q, Shi YH, et al. CHL1 is involved in human breast tumorigenesis and progression. Biochem Biophys Res Commun. Aug 23 2013;438(2):433-438.

48. Senchenko VN, Krasnov GS, Dmitriev AA, et al. Differential expression of CHL1 gene during development of major human cancers. PLoS One. Mar 7 2011;6(3):e15612.

49. Chen J, Jiang C, Fu L, et al. CHL1 suppresses tumor growth and metastasis in nasopharyngeal carcinoma by repressing PI3K/AKT signaling pathway via interaction with Integrin $\beta 1$ and Merlin. Int J Biol Sci. 2019;15(9):1802-1815.

50. Martín-Sánchez E, Mendaza S, Ulazia-Garmendia A, et al. CHL1 hypermethylation as a potential biomarker of poor prognosis in breast cancer. Oncotarget. Feb 28 2017;8(9):15789-15801.

51. Thie M, Denker HW. In vitro studies on endometrial adhesiveness for trophoblast: cellular dynamics in uterine epithelial cells. Cells Tissues Organs. 2002;172(3):237-252.

\section{Figure legends}

Fig.1 miR-1271-5p is downregulated in serum and placenta tissues of PE. (A) qRT-PCR analysis was used to examine miR-1271-5p in serum of PE patients. U6 was used as the control. ${ }^{*} P<0.05$, vs. normal pregnancy. (B) qRT-PCR analysis examined miR-1271-5p expression in PE placentas tissue $(n=30)$ and normal pregnancy placentas tissue $(n=30)$. Normalized to U6. $* * P<0.01$, vs. normal pregnancy placentas. 
Fig.2 miR-1271-5p inhibits trophoblast cell proliferation and invasion.

(A and B) The expression of miR-1271-5p was detected by using qRT-PCR in HTR8/SVneo cells after transfecting with miR-1271-5p mimic or inhibitor or their control RNA. $* * P<0.01$, $* * * P<0.001$ vs. corresponding control. (C) Cell viability was detected by MTS assay in HTR-8/SVneo cells transfected with miR-1271-5p mimics or inhibitor or their control RNA. $* P<0.05$ vs. corresponding control. (D and E) HTR8/SVneo cells were prepared as in (A), and cell invasion ability was examined by transwell assays. ${ }^{*} P<0.05$ vs. corresponding control. (F and G) HTR8/SVneo cells were prepared as in (B), cell invasion ability was examined by transwell assays. $* * P<0.01$ vs. corresponding control. Bar=10 $\mu \mathrm{m}$. Data shown are mean \pm standard deviation of three independent experiments.

Fig.3 miR-1271-5p regulates Grhl2 expression by targeting the 3'-UTR of Grhl2. (A) Venn diagram hybrid of mRNA from three different target prediction programs. The result showed there are 66 potential genes of miR-1271-5p. Among them, only 11 candidates have been reported with a function of cell proliferation and invasion. (B) miR-1271-5p mimic or inhibitor or their negative controls was transfected into HTR8/SVneo cells and RT-qPCR analysis was used to determine the indicated genes mRNA expression. $* P<0.05$ vs. corresponding control. (C) Prediction of miR-1271-5p binding site in Grhl2 3'-UTR. (D) Luciferase reporter assays were performed to detect the luciferase activity in HTR8/SVneo cells after co-transfected with the miR-1271-5p mimic and Grhl2 3'-UTR-luciferase reporter (WT/mut). ${ }^{* *} P<0.01$ vs. mimic-NC. (E) HTR8/SVneo cells were transfected with indicated miR-1271-5p RNA and western blot analysis was used to detect Grhl2 protein expression. (F) Quantitative analysis of (E). $* * P<0.01, * * * P<0.001$ vs. corresponding control. (G-I) qRT-PCR and western blot analysis was used to detect Grhl2 expression in primary trophoblast cellsafter transfecting miR-1271-5p mimic or inhibitor or their corresponding control RNA. $* P<0.05, * * P<0.01$ vs. corresponding control.

Fig. 4 Grhl2 overexpression inhibits the proliferation and migration of trophoblast cell. (A-C) trophoblast cell were transfected with pcDNA3.1-Grhl2 (Grhl2) or pcDNA3.1 control vector (Vector); then, RT-qPCR and western blot detected the mRNA and protein expression of Grhl2. $* * \mathrm{P}<0.01,{ }^{*} * * \mathrm{P}<0.001$ vs. vector. (D) Trophoblast cell were transfected with Grhl 2 or control vector; then, MTS assay detected cell proliferation. $* \mathrm{P}<0.05$ vs. Vector. (E) Trophoblast cell were treated as above, and wound-scratch healing assay detected cell migration. (F) Quantitative analysis of (E). Data are expressed as mean \pm SEM from three independent experiments. ${ }^{*} \mathrm{P}<0.05$ vs. control. 
Fig. $5 \mathrm{miR}-1271-5 \mathrm{p} / \mathrm{Grhl} 2$ axis regulates trophoblast cell invasion and proliferation (A) qRT-PCR analysis was performed to examine the mRNA expression of Grhl2 in PE placentas and normal pregnancy placentas. ${ }^{*} P<0.05$ vs. normal pregnancy placentas. (B) The relationship between miR-1271-5p expression and Grhl2 mRNA expression in PE placentas was analyzed by Spearman's correlation analysis $(\mathrm{R}=-0.03566, P=0.0451)$. (C and $\mathrm{D})$ HTR8/SVneo cells were transfected with miR-1271-5p mimic or control mimic, respectively, or co-transfected with pcDNA3.1-Grhl2 or empty vector. Cell invasion ability was evaluated by transwell assay. Bar=10 $\mu \mathrm{m} .{ }^{*} P<0.05$ vs. corresponding control. (E) HTR8/SVneo cells were transfected with RNA or construct as above and cell viability was detected by MTS assay. $* P<0.05, * * P<0.01$ vs. corresponding control.

Fig. 6 Grhl2 promotes the transcription of CHL1 and mediates miR-1271-5p-regulated trophoblast cell invasion and proliferation

(A) HTR8/SVneo cells were transfected with miR-1271-5p mimic or control mimic, respectively, or co-transfected with pcDNA3.1-Grhl2 or empty vector. And then western blot was used to detect the CHL1 and $\beta$-catenin protein level. (B) Quantitative analysis of (A). Data are expressed as mean \pm SEM from three independent experiments. $* * \mathrm{P}<0.01$, *** $\mathrm{P}<$ 0.001 vs. corresponding control. (C) There are 3 putative binding elements within the $2-\mathrm{kb}$ promoter region of CHL1. (D) CHL1 promoter luciferase reporters were cotransfected along with the Grhl2 expression vector or miR-1271-5p into trophoblast cell and luciferase reporter assays were performed. $* \mathrm{P}<0.05$, vs. corresponding control. (E) ChIP-qPCR was used to detect the binding of Grhl2 to the CHL1 promoter region in trophoblast cell. Arrowheads indicate the position of primers used for ChIP-PCR. $*$ P $<0.05$ vs. IgG. (E) HTR8/SVneo cells were transfected with indicated construct and cell viability was detected by MTS assay. ${ }^{*} P<$ $0.05, * * P<0.01$ vs. corresponding control. 
Figures

A

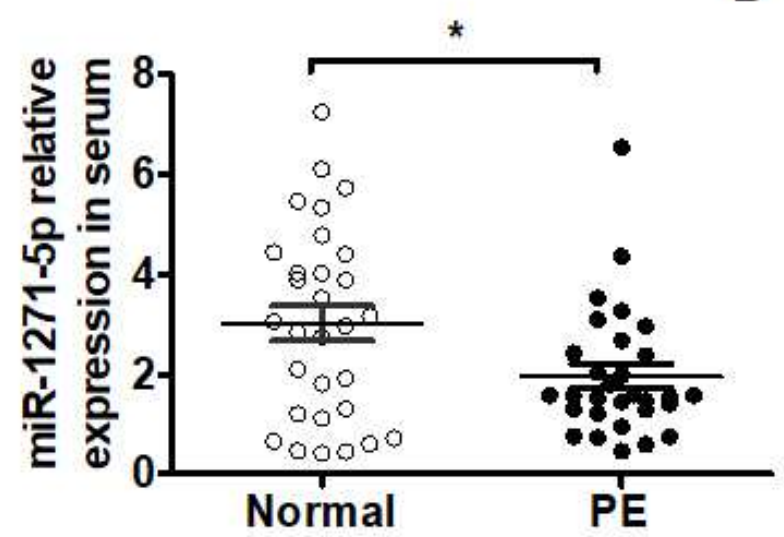

B

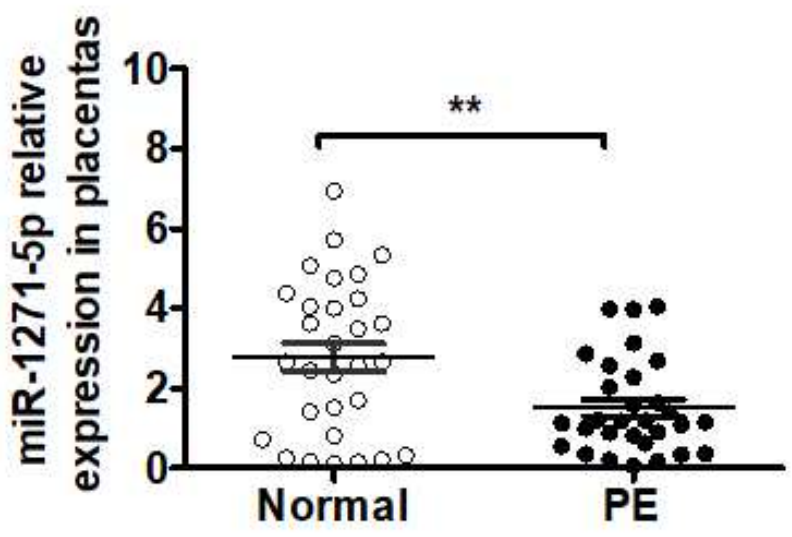

Figure 1

miR-1271-5p is downregulated in serum and placenta tissues of PE. (A) qRT-PCR analysis was used to examine miR-1271-5p in serum of PE patients. U6 was used as the control. ${ }^{*} P<0.05$, vs. normal pregnancy. (B) qRT-PCR analysis examined miR-1271-5p expression in PE placentas tissue $(n=30)$ and normal pregnancy placentas tissue $(n=30)$. Normalized to U6. ${ }^{\star *} P<0.01$, vs. normal pregnancy placentas. 

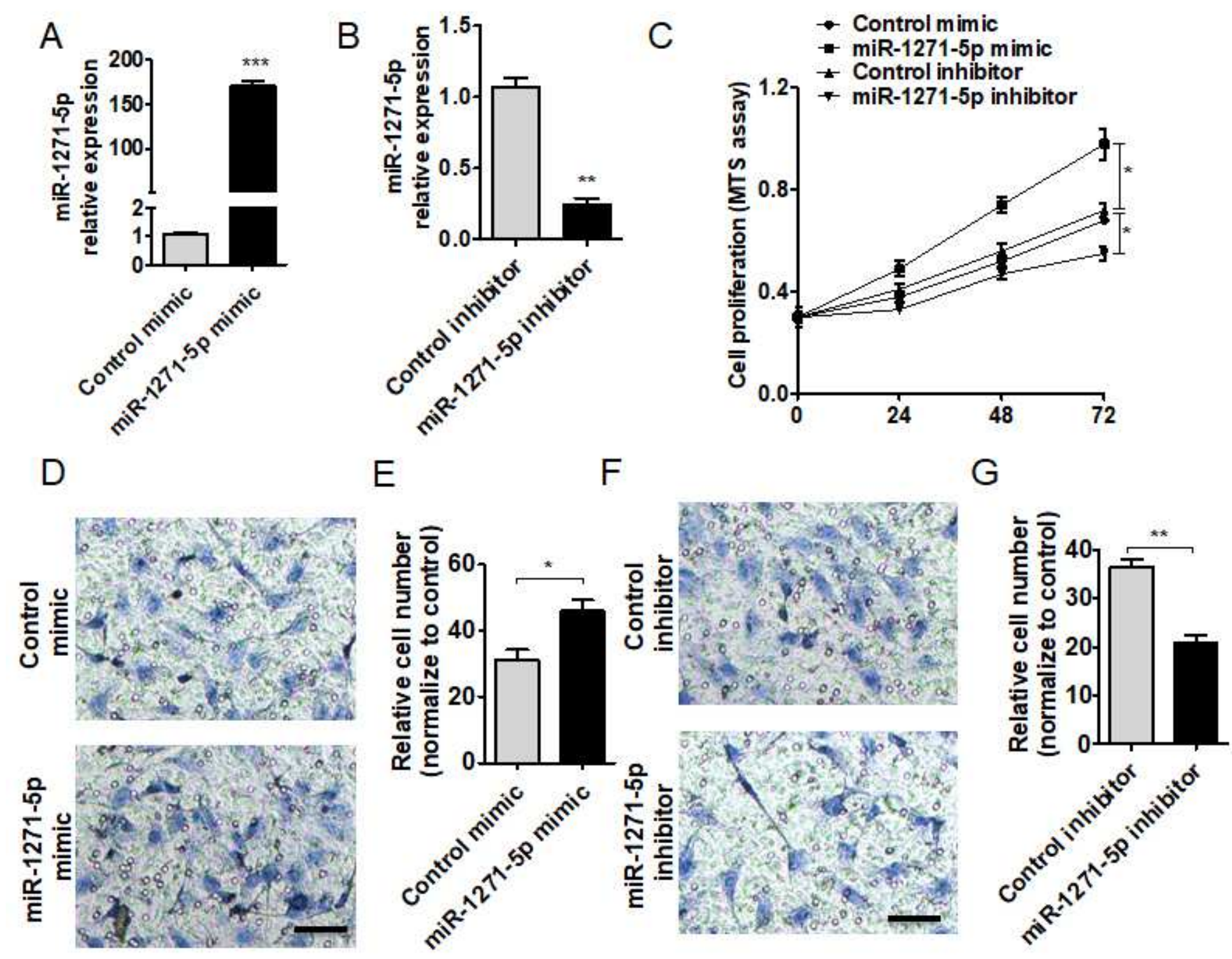

Figure 2

miR-1271-5p inhibits trophoblast cell proliferation and invasion. ( $A$ and $B$ ) The expression of miR-1271-5p was detected by using qRT-PCR in HTR8/SVneo cells after transfecting with miR-1271-5p mimic or inhibitor or their control RNA. ${ }^{*} \mathrm{P}<0.01,{ }^{\star * \star} \mathrm{P}<0.001$ vs. corresponding control. (C) Cell viability was detected by MTS assay in HTR-8/SVneo cells transfected with miR-1271-5p mimics or inhibitor or their control RNA. ${ }^{*} P<0.05$ vs. corresponding control. ( $D$ and $E$ ) HTR8/SVneo cells were prepared as in (A), and cell invasion ability was examined by transwell assays. ${ }^{*} \mathrm{P}<0.05$ vs. corresponding control. ( $\mathrm{F}$ and $\left.\mathrm{G}\right)$ HTR8/SVneo cells were prepared as in (B), cell invasion ability was examined by transwell assays. ${ }^{*} \mathrm{P}<$ 0.01 vs. corresponding control. Bar $=10 \mu \mathrm{m}$. Data shown are mean \pm standard deviation of three independent experiments. 
A
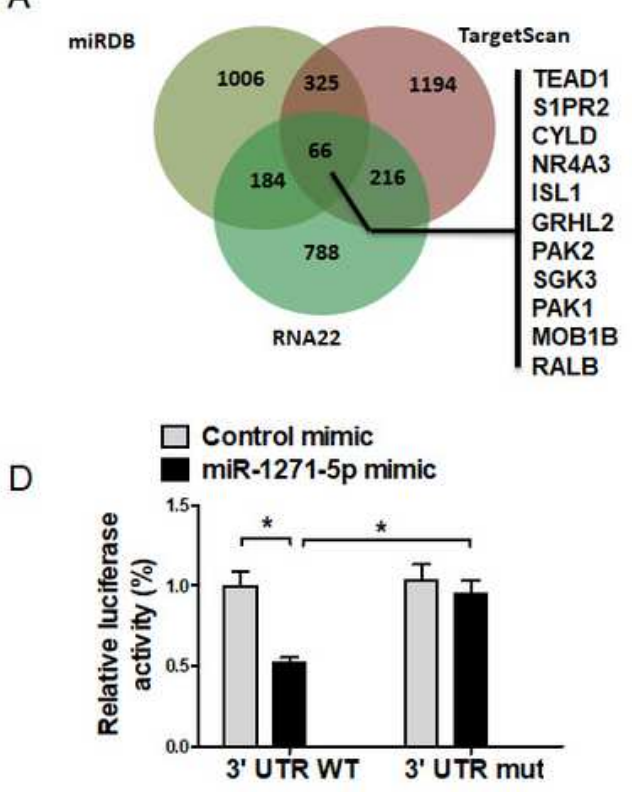

$E$

Grhl2

$\beta$-actin

$$
\text { HTR-8/SVneo }
$$

G

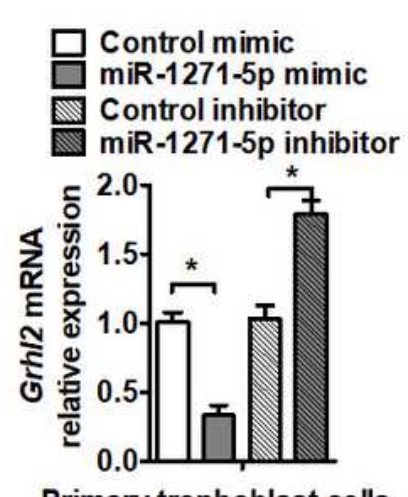

Primary trophoblast cells

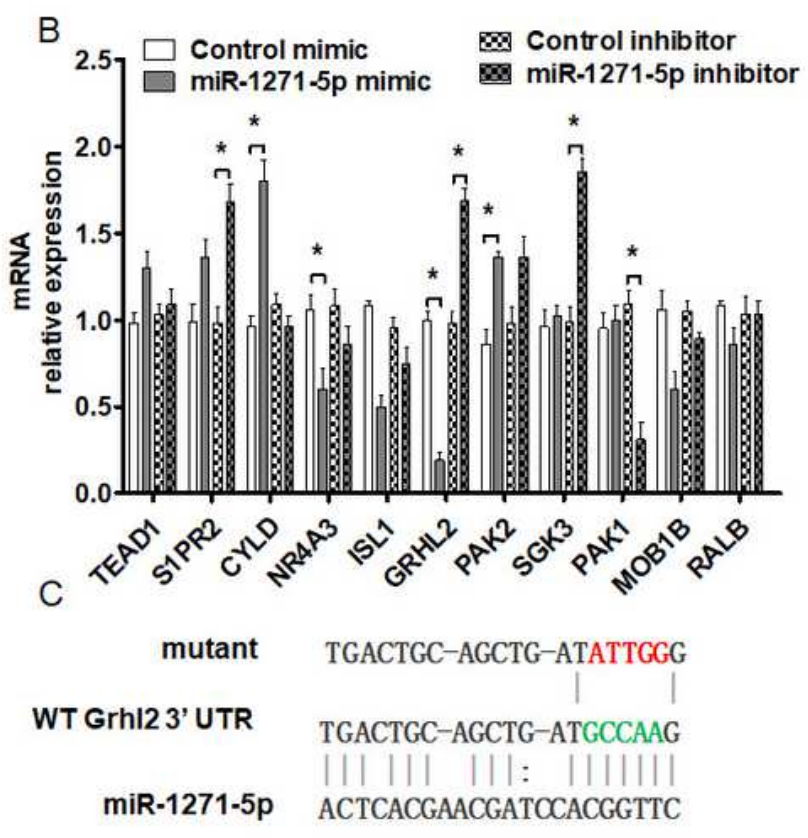

$\mathrm{F}$

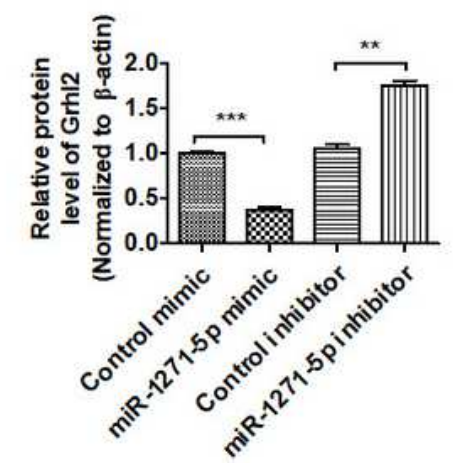

$\mathrm{H}$

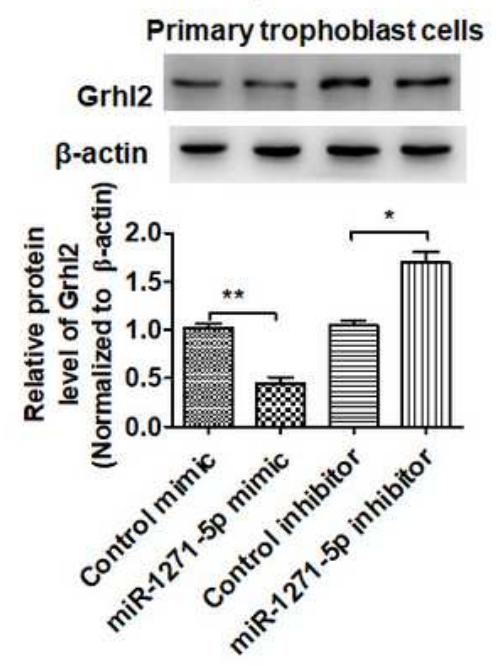

\section{Figure 3}

miR-1271-5p regulates Grhl2 expression by targeting the 3'-UTR of Grhl2. (A) Venn diagram hybrid of mRNA from three different target prediction programs. The result showed there are 66 potential genes of miR-1271-5p. Among them, only 11 candidates have been reported with a function of cell proliferation and invasion. (B) miR-1271-5p mimic or inhibitor or their negative controls was transfected into HTR8/SVneo cells and RT-qPCR analysis was used to determine the indicated genes mRNA expression. 
${ }^{*} \mathrm{P}<0.05$ vs. corresponding control. (C) Prediction of miR-1271-5p binding site in Grhl2 3'-UTR. (D) Luciferase reporter assays were performed to detect the luciferase activity in HTR8/SVneo cells after cotransfected with the miR-1271-5p mimic and Grhl2 3'-UTR-luciferase reporter (WT/mut). ${ }^{\star \star} \mathrm{P}<0.01 \mathrm{vs}$. mimic-NC. (E) HTR8/SVneo cells were transfected with indicated miR-1271-5p RNA and western blot analysis was used to detect Grhl2 protein expression. (F) Quantitative analysis of (E). ${ }^{\star *} P<0.01,{ }^{*} * \mathrm{P}<$ 0.001 vs. corresponding control. (G-I) qRT-PCR and western blot analysis was used to detect Grhl2 expression in primary trophoblast cellsafter transfecting miR-1271-5p mimic or inhibitor or their corresponding control RNA. ${ }^{*} \mathrm{P}<0.05,{ }^{*} \mathrm{P}<0.01$ vs. corresponding control.

A

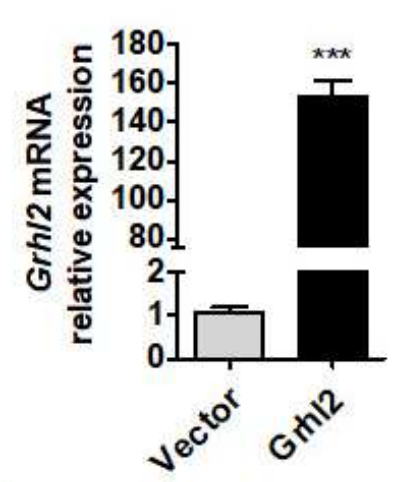

E

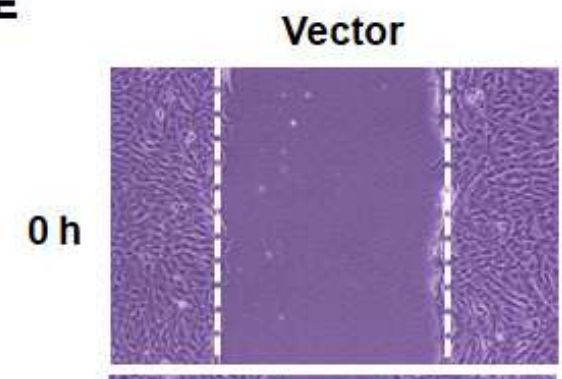

B

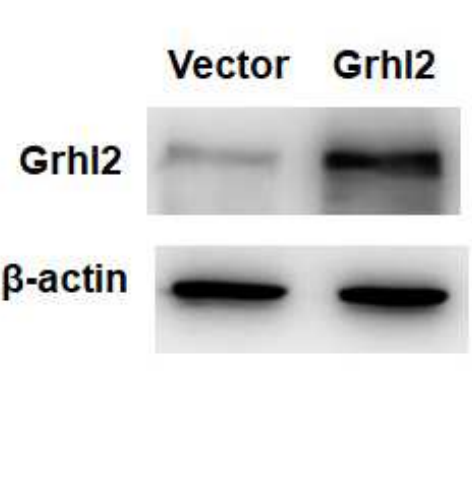

C Grhl2

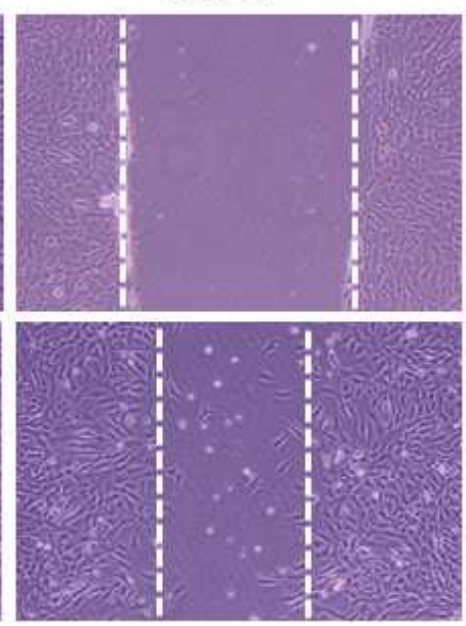

D

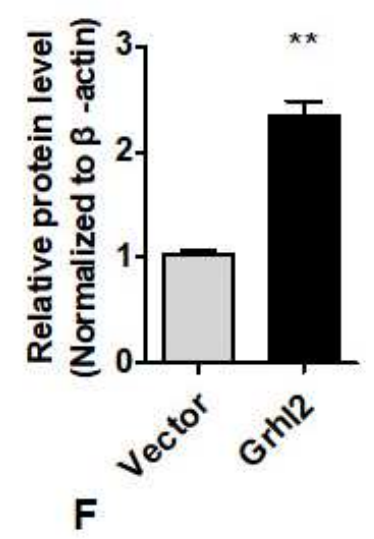

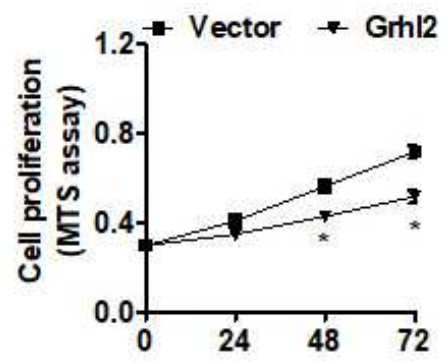

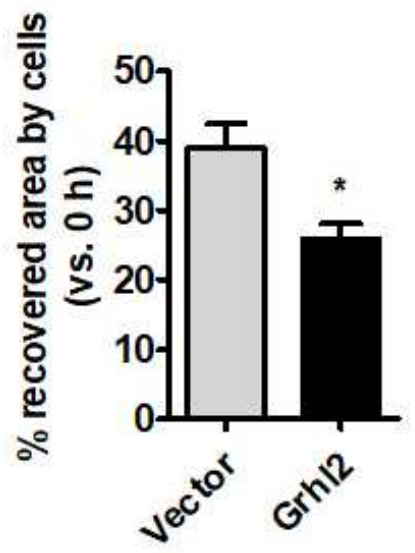

Figure 4

Grhl2 overexpression inhibits the proliferation and migration of trophoblast cell. (A-C) trophoblast cell were transfected with pcDNA3.1-Grhl2 (Grhl2) or pcDNA3.1 control vector (Vector); then, RT-qPCR and western blot detected the mRNA and protein expression of Grhl2. ${ }^{* \star P}<0.01,{ }^{\star} * \star P<0.001$ vs. vector. (D) Trophoblast cell were transfected with Grhl2 or control vector; then, MTS assay detected cell proliferation. ${ }^{*} \mathrm{P}<0.05$ vs. Vector. (E) Trophoblast cell were treated as above, and wound-scratch healing assay detected cell migration. (F) Quantitative analysis of $(E)$. Data are expressed as mean \pm SEM from three independent experiments. ${ }^{*} \mathrm{P}<0.05$ vs. control. 17 
A

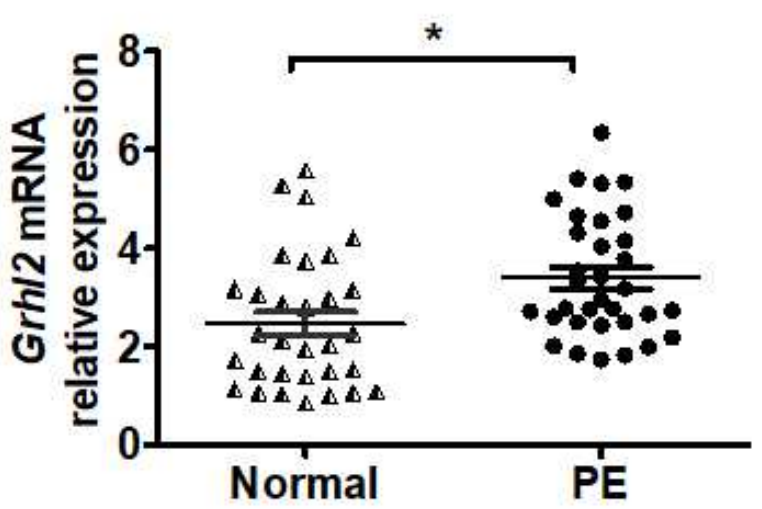

C
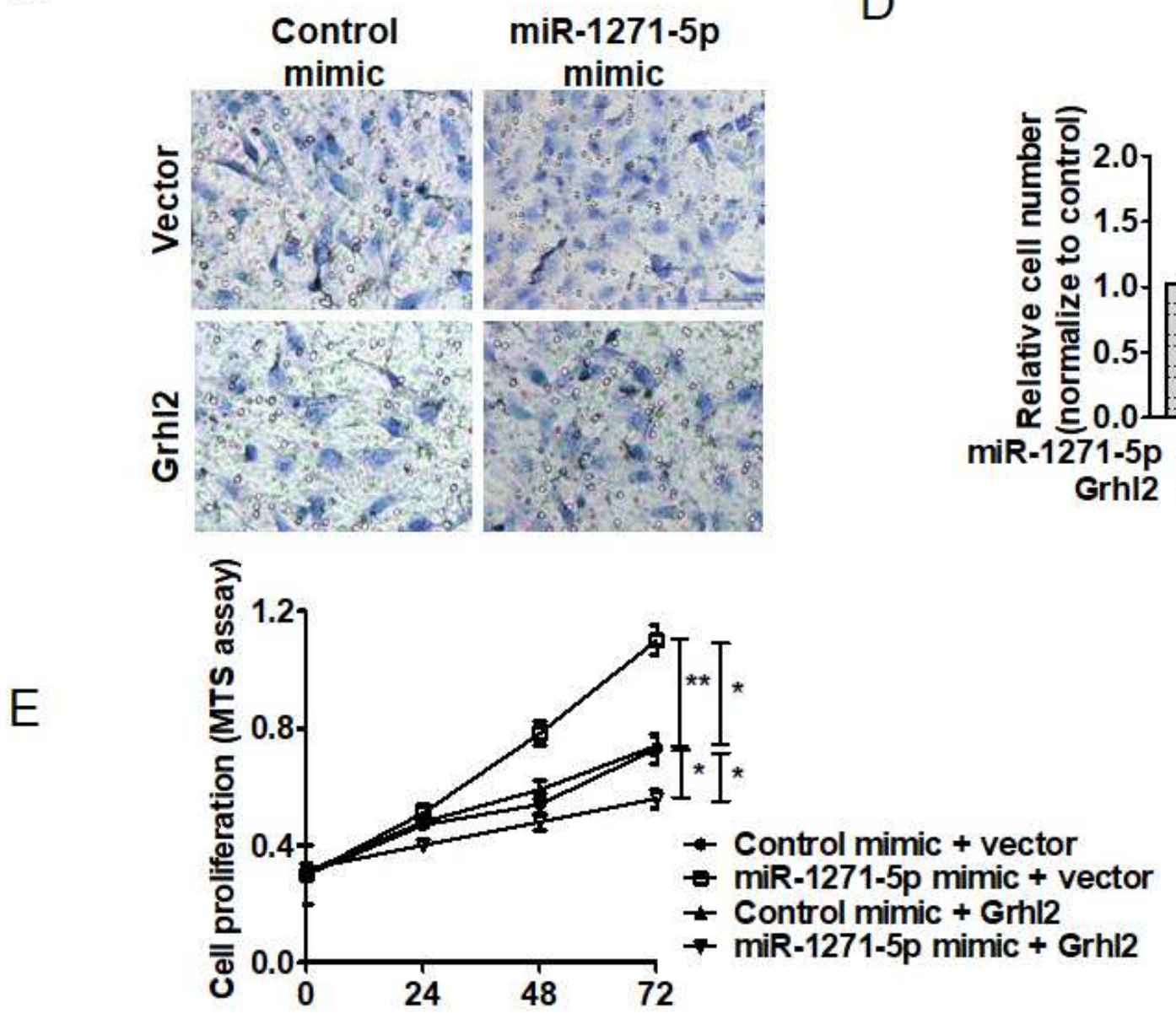

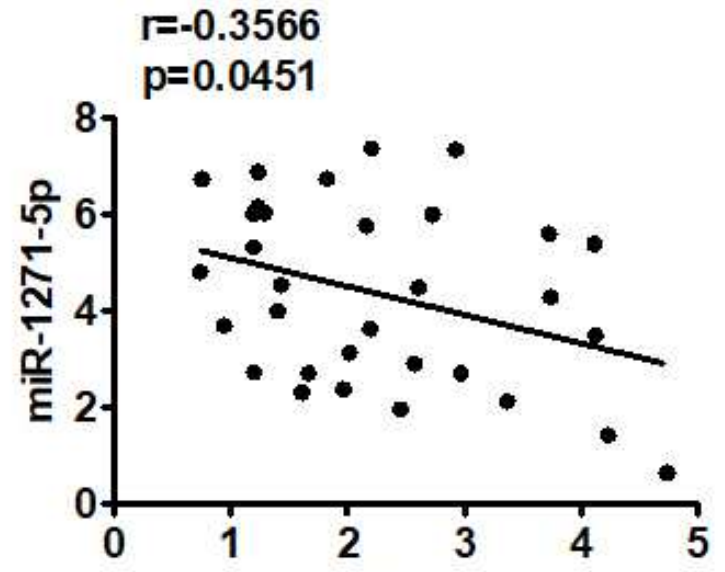

Grhl2

Figure 5

miR-1271-5p/Grhl2 axis regulates trophoblast cell invasion and proliferation (A) qRT-PCR analysis was performed to examine the mRNA expression of Grhl2 in PE placentas and normal pregnancy placentas. ${ }^{*} \mathrm{P}<0.05$ vs. normal pregnancy placentas. (B) The relationship between miR-1271-5p expression and Grhl2 mRNA expression in PE placentas was analyzed by Spearman's correlation analysis $(R=-0.03566$, $P=0.0451$ ). ( $C$ and D) HTR8/SVneo cells were transfected with miR-1271-5p mimic or control mimic, respectively, or co-transfected with pcDNA3.1-Grhl2 or empty vector. Cell invasion ability was evaluated 
by transwell assay. Bar=10 $\mu \mathrm{m}$. ${ }^{*} \mathrm{P}<0.05$ vs. corresponding control. (E) HTR8/SVneo cells were transfected with RNA or construct as above and cell viability was detected by MTS assay. ${ }^{*} \mathrm{P}<0.05, * \star \mathrm{P}<$ 0.01 vs. corresponding control.

A

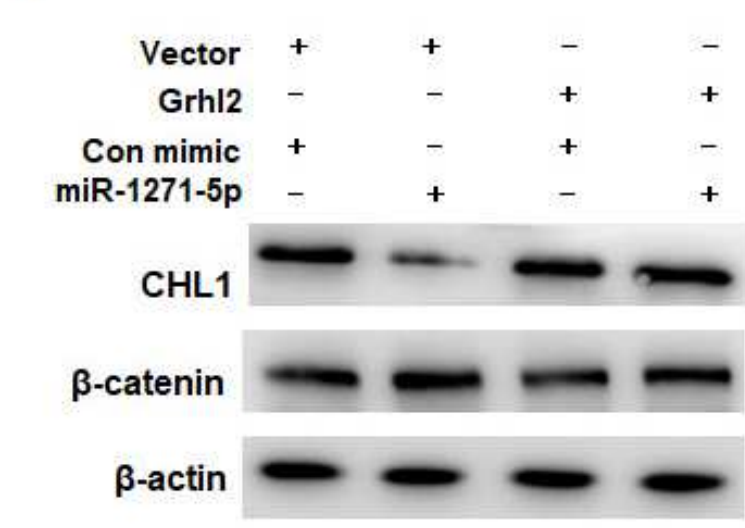

C

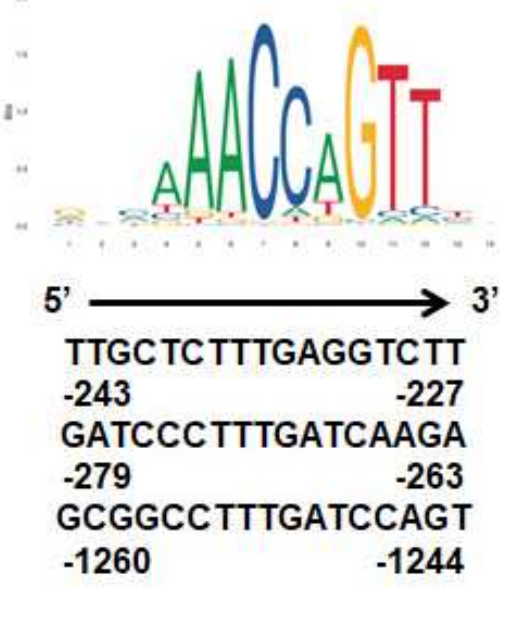

E
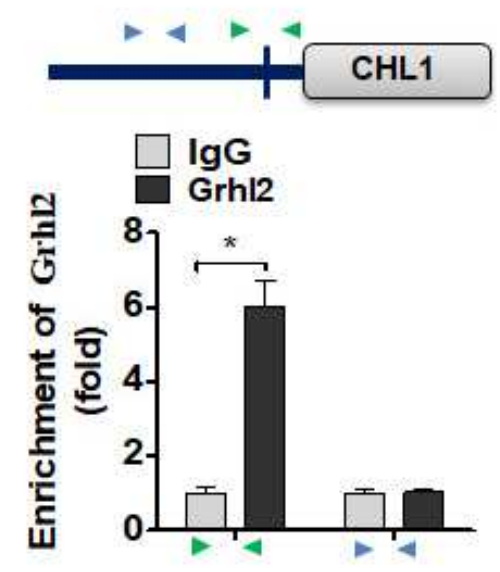

B
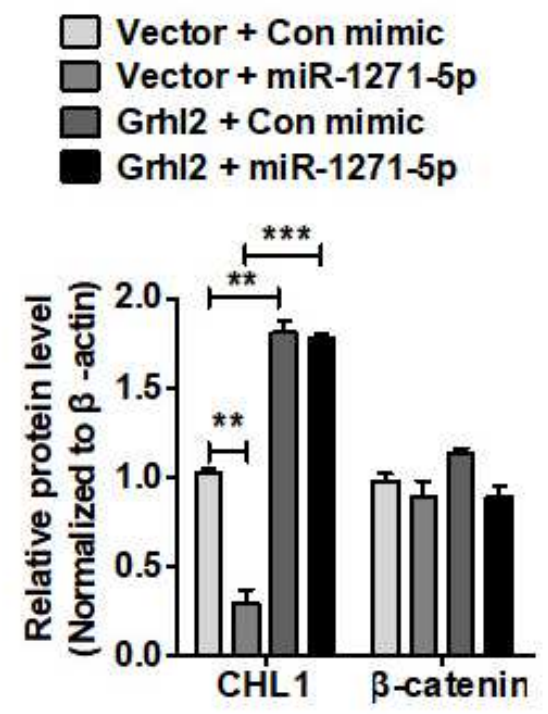

D
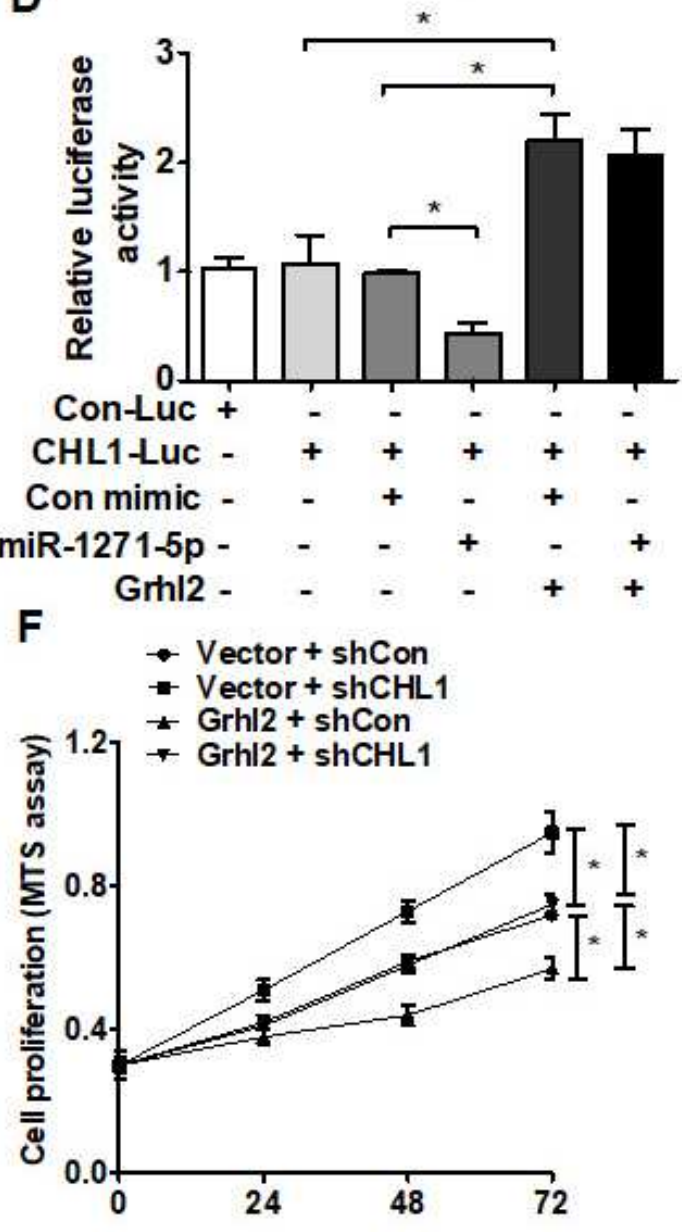

Figure 6

Grhl2 promotes the transcription of CHL1 and mediates miR-1271-5p-regulated trophoblast cell invasion and proliferation (A) HTR8/SVneo cells were transfected with miR-1271-5p mimic or control mimic, 
respectively, or co-transfected with pcDNA3.1-Grhl2 or empty vector. And then western blot was used to detect the $\mathrm{CHL} 1$ and $\beta$-catenin protein level. (B) Quantitative analysis of (A). Data are expressed as mean \pm SEM from three independent experiments. ${ }^{\star *} \mathrm{P}<0.01$, ${ }^{\star * *} \mathrm{P}<0.001 \mathrm{vs}$. corresponding control. (C) There are 3 putative binding elements within the 2-kb promoter region of CHL1. (D) CHL1 promoter luciferase reporters were cotransfected along with the Grhl2 expression vector or miR-1271-5p into trophoblast cell and luciferase reporter assays were performed. ${ }^{*} P<0.05$, vs. corresponding control. (E) ChIP-qPCR was used to detect the binding of Grhl2 to the $\mathrm{CHL} 1$ promoter region in trophoblast cell. Arrowheads indicate the position of primers used for ChIP-PCR. ${ }^{*} P<0.05$ vs. IgG. (E) HTR8/SVneo cells were transfected with indicated construct and cell viability was detected by MTS assay. ${ }^{\star} \mathrm{P}<0.05,{ }^{\star *} \mathrm{P}<0.01$ vs. corresponding control.

\section{Supplementary Files}

This is a list of supplementary files associated with this preprint. Click to download.

- SupplementaryOriginalData.pdf 